\title{
GLOBAL MAPPING PROJECT - APPLICATIONS AND DEVELOPMENT OF VERSION 2 DATASET
}

\author{
T. Ubukawa, T. Nakamura, T. Otsuka, T. Iimura, N. Kishimoto, K. Nakaminami, \\ Y. Motojima, M. Suga, Y. Yatabe, M. Koarai, T. Okatani
}

Geospatial Information Authority of Japan, 1 Kitasato, Tsukuba, Ibaraki 305-0811, Japan, env-gm@gsi.go.jp

KEY WORDS: Global Map, Environment, International Cooperation, ISCGM, Framework data, National Mapping Organization

\begin{abstract}
:
The Global Mapping Project aims to develop basic geospatial information of the whole land area of the globe, named Global Map, through the cooperation of National Mapping Organizations (NMOs) around the world. The Global Map data can be a base of global geospatial infrastructure and is composed of eight layers: Boundaries, Drainage, Transportation, Population Centers, Elevation, Land Use, Land Cover and Vegetation.

The Global Map Version 1 was released in 2008, and the Version 2 will be released in 2013 as the data are to be updated every five years. In 2009, the International Steering Committee for Global Mapping (ISCGM) adopted new Specifications to develop the Global Map Version 2 with a change of its format so that it is compatible with the international standards, namely ISO 19136 and ISO 19115. With the support of the secretariat of ISCGM, the project participating countries are accelerating their data development toward the completion of the global coverage in 2013, while some countries have already released their Global Map version 2 datasets since 2010 .

Global Map data are available from the Internet free of charge for non-commercial purposes, which can be used to predict, assess, prepare for and cope with global issues by combining with other spatial data. There are a lot of Global Map applications in various fields, and further utilization of Global Map is expected. This paper summarises the activities toward the development of the Global Map Version 2 as well as some examples of the Global Map applications in various fields.
\end{abstract}

\section{INTRODUCTION}

\subsection{Background and History of Global Mapping Project}

The Global Mapping Project is an endeavour to develop basic geospatial dataset, named "Global Map", in a consistent manner In 1992, the United Nations Conference on Environment and Development, known as "Earth Summit", was held in Rio de Janeiro where the importance of various kinds of information for decision-making, including geospatial information, was recognized. In response to this, the Ministry of Construction (the current Ministry of Land, Infrastructure, Transport and Tourism; MLIT) of Japan proposed the concept of the Global Mapping Project in 1992, and the Global Mapping Project was launched in 1996 with the establishment of the International Steering Committee for Global Mapping (ISCGM). Since then, the National Mapping Organizations (NMOs) of more than 180 countries and regions around the world participate in the project and they have been working to develop their Global Map datasets through international cooperation. The ISCGM, consists of 20 members, has promoted the Global Mapping Project as the steering body of the project.

\subsection{Steering Body}

The ISCGM, consists of 20 members including 17 heads of NMOs and is chaired by Professor D. R. Fraser Taylor of Carlton University, Canada, has conducted and managed the project. The Geospatial Information Authority of Japan (GSI) serves as the secretariat of the ISCGM. The ISCGM holds an annual meeting to achieve its primary purpose, i.e., to examine measures that concerned national, regional and international organizations can take to foster the development of Global Map in order to facilitate the implementation of global agreements and conventions for environmental protection as well as the mitigation of natural disasters and to encourage economic growth within the context of sustainable development.

The ISCGM is also actively participating in various international activities in the field of geospatial information, such as ISO/TC 211 and GGIM as an external liaison or a partner organization.

\subsection{Participants}

A unique character of the Global Mapping Project is voluntary participations of NMOs around the world. This enables to collect reliable data from each country. With the recommendation of the Director of the UN Statistics Division, ISCGM invited NMOs of all over the world for their participation in 1998, which resulted in the great increase of participants. Currently, as of April 2012, 165 countries and 16 regions participate in the project.

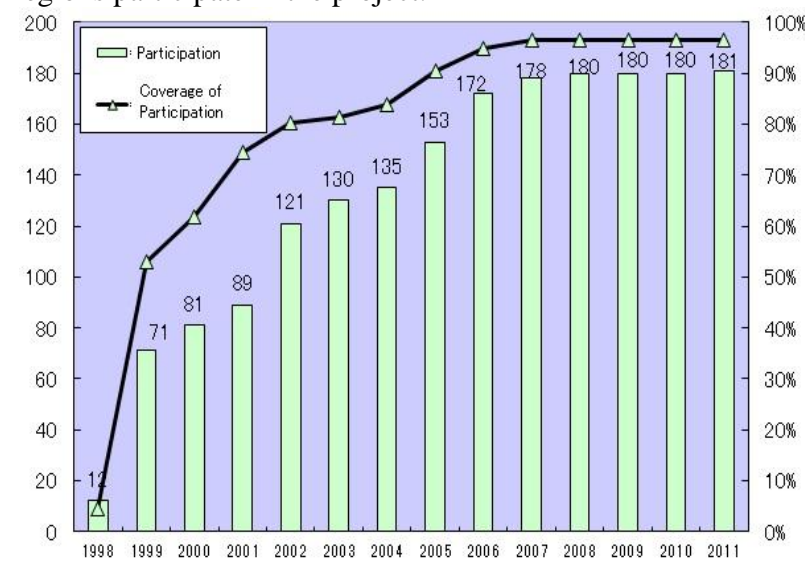

Figure 1: Number of the participating countries/regions 


\subsection{Global Map Dataset}

Outline of the dataset: The Global Map is digital geospatial dataset of the whole land area of the globe at a scale of 1 to $1,000,000$ (or in 30 arc seconds resolution) and has eight thematic layers, namely, boundaries, drainage, population centers, transportation, elevation, land use, land cover, and vegetation (percent tree cover). The former four layers are in vector format, while the latter four layers are in raster. The data are stored in the geographic coordinate with the GRS80 ellipsoid and the ITRF94 system. All the data are developed on the basis of the Global Map Specifications whose latest version is 2.1 adopted in 2011. The vector layers are made available both in GML and shape file formats, and raster layers are in the BIL and TIFF formats.

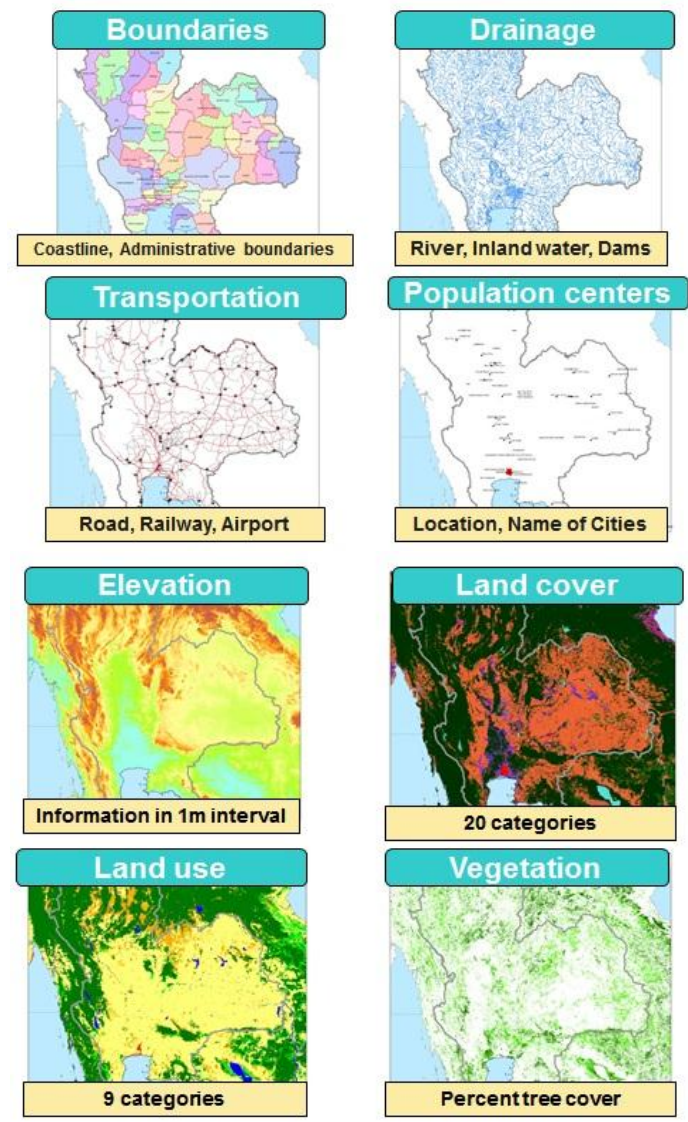

Figure 2: Eight layers of Global Map

Progress: As of April 1, 2012, the Global Map datasets of 79 countries/regions have been released. Furthermore, the Global Land Cover and Vegetation, created by using MODIS data observed in 2003, were released in 2008 as part of Global Map Version 1.

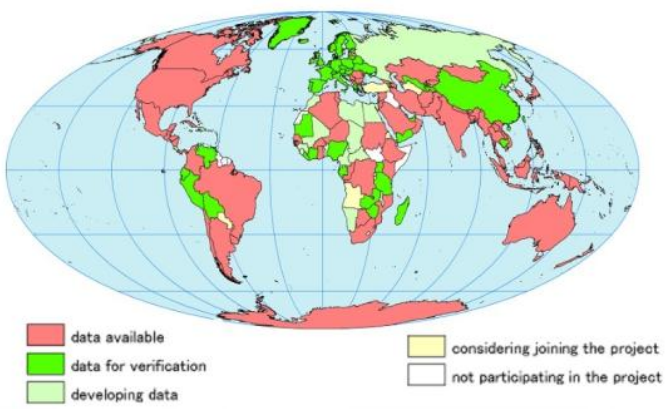

Figure 3: Progress of Data Development
The development of the Global Map Version 1 was terminated in 2008 with the release of the Global Map Version 1 datasets of more than 70 countries/regions, the Global Land Cover and the Global Vegetation. As the Global Map is to be updated every five years to monitor the changes of the environment, the NMOs have been working to develop the Global Map Version 2 by 2013 .

Data policy: Although all participating countries share the basic policy, i.e., the Global Map data are released free of charge for non-commercial purposes, the data policies of Global Map datasets differ from country to country as they are determined by NMOs that have developed the data. Each dataset is subject to copy right protection. The data users confirm data policy when they download the data from the Internet.

\section{DEVELOPMENT OF GLOBAL MAP DATA}

\subsection{Global Map Specifications and Their Revision}

Global Map Specifications: The Global Map Specifications define the Global Map itself, including the structure, attributes, metadata and format of the dataset, which give common understanding of the dataset to the data users and developers. The Specifications have been used to develop Global Map datasets of many countries after the first release in 2000.

Revision of the Specifications: Since some parts needed to be updated after the development of the Global Map Version 1 based on the specification Version 1, the Global Map Specifications were revised and updated to Version 2 in 2009. For the revision, ISCGM Secretariat conducted several surveys and held a workshop to collect comments from users and NMOs. The main points of the revision were the followings:

1) Modifications of the Data Dictionary,

2) Change of vector format from VPF to GML (ISO19136),

3) Change of file coverage to be provided by countries, and

4) Change of metadata format to be compliant to ISO19115.

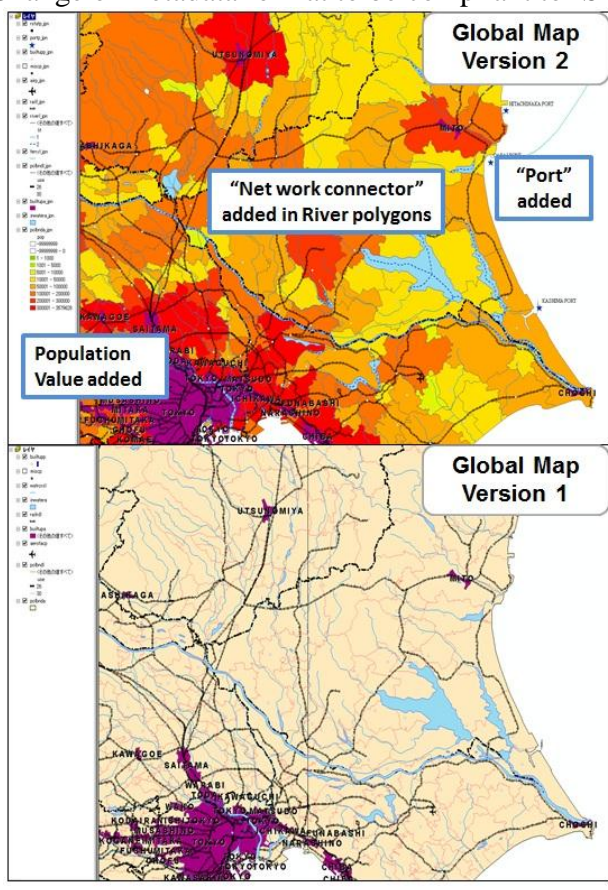

Figure 4: Changes in Global Map Version 2 
Although it seems that the international standards have not yet become "de facto" standards on GIS format, the ISCGM tried to be vendor-neutral and compliant with the existing international standards, which could be a good practice shared with the participating NMOs. The application schema which describes Global Map Vector format and the Global Map Metadata Schema are described in the Global Map Specifications.

\subsection{Data Developing Schema and Methods}

Data Developing Schema: The project participating NMOs are expected to develop their own Global Map datasets. However, if some countries have difficulties in developing their Global Maps, such as lack of technical equipment and financial limitation, other countries voluntarily support these countries to develop their data. The ISCGM manages and promotes the data development and the secretariat checks the data so that the data can satisfy the minimum requirements mentioned in the Global Map Specifications.

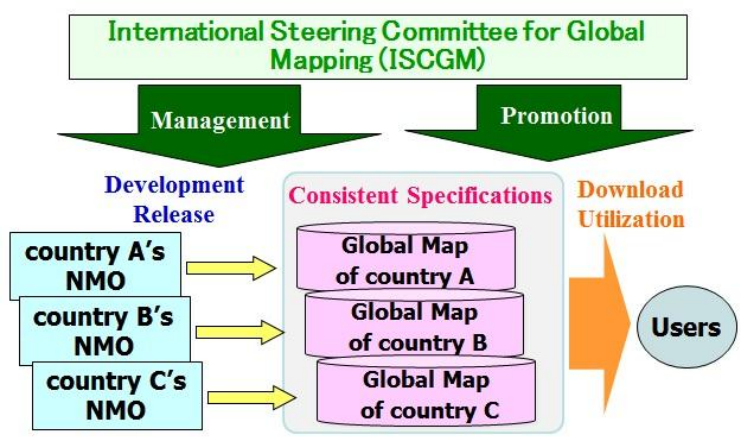

Figure 5: Framework of the project

Data Developing Methods: The sources of the Global Map can vary from country to country. In a case that NMOs have some GIS datasets suitable for Global Map, they generally convert them and reclassify the data into Global Map structure. If NMOs have no digital data, they usually develop their data by digitizing the exiting small scale maps that NMOs own. Other existing global data, such as VMAP-0 and satellite images can be used where there is no appropriate data available as the source. Although the Global Map Specifications define the minimum requirements for data quality, the quality of each data is not always at the same level. Its metadata, which is concordant with ISO19115, shows the source of each Global Map dataset and explains about the quality.

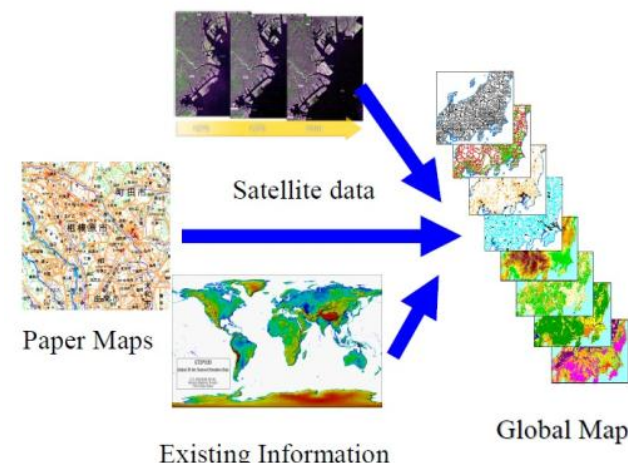

Figure 6: Data development flow

To give a certain guideline to develop the data, the secretariat of the ISCGM developed the Manual for Development and Revision of Global Map, which includes data processing methods from several kinds of materials, such as printed maps and digital geospatial information. It also mentions about the data development method using satellite images based on the lessons learnt from some preliminary effort of the ISCGM Secretariat. The manual also recommended that participants use best available data because the data sources directly affect the quality of the product.

\subsection{Quality Control}

Global Map Data Check Software: In order to improve the quality of the data toward the completion of the Global Map Version 2, GSI has developed the Global Map Data Check software (GMDC) from 2010 to 2011, the latest version of which is 2.0. This software was specially dedicated to the Global Map and distributed to the project participating NMOs. By using this software, each NMO easily finds topological errors, inappropriate attributes and logical inconsistency of features, which they had to manually detect before. The GMDC detects basic errors, such as dangles, overlap and disconnection within a feature class. In addition, it also detects logical errors between feature classes such as the relation between the boundary line and the boundary areas. Major functions of the GMDC are as follows:

1) Import GML/shape file data,

2) Overlay images (tiff/bil),

3) Basic check (file name, attributes, topology and so on),

4) Inter-feature check

5) Display/hide error marks and save the error list, and

6) Modify errors automatically or manually.

\section{Extremely Short Line/Segment Contact within}
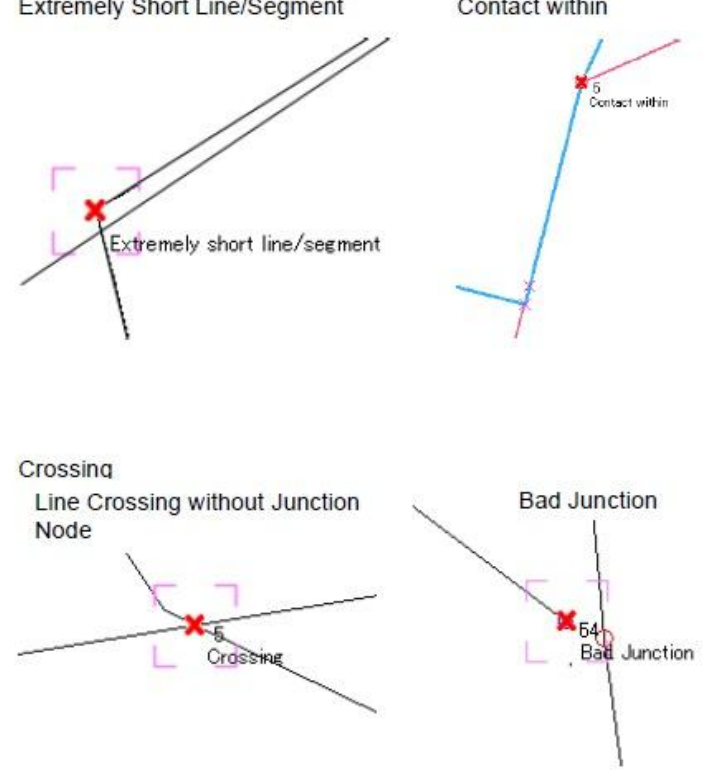

Figure 7: Examples of errors detected by GMDC

The GMDC enables participating NMOs to modify their data easily even if they do not have enough number of GIS software licenses. It is expected to play an important role in developing Global Map dataset especially in the countries whose GIS environment is not sufficient.

\subsection{Capacity Building}

The Global Mapping Project also plays a role in capacity building. GSI annually hosts a two and half month group training course on the development of Global Map conducted by the Japan International Cooperation Agency (JICA). This 
training mainly focuses on learning data developing methods, such as GIS, remote sensing, spatial data infrastructure and international standards and is targeted to the technical staff of NMOs. From 1994 to 2011, 106 participants from 60 countries completed the program, having developed Global Map of 43 countries.

\section{APPLICATION}

\subsection{Application Cases}

Applications in the field of disaster mitigation: To prevent secondary disaster and to efficiently provide aids to victims in the event of a large scale natural disaster, GSI prepares maps by using Global Map data, which show geographic features of disaster hit area. These maps are distributed to all over the world through the Relief Web (www.reliefweb.int) operated by the United Nations Office for the Coordination of Humanitarian Affairs (UNOCHA) as well as the websites of the ISCGM and GSI.

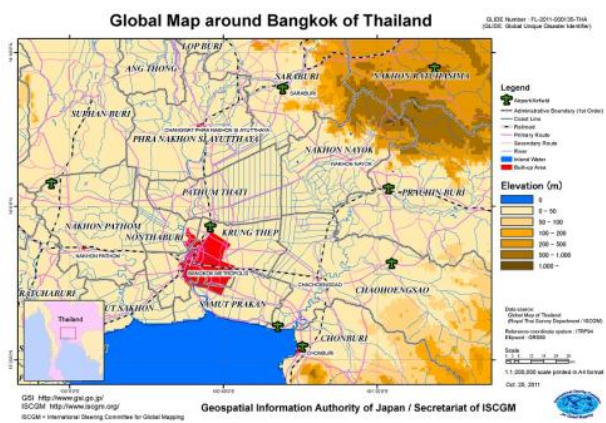

Figure 8: Global Map around Bangkok, Thailand (2011)

Applications in the flood analysis: Global Map data are used for runoff analysis in the Integrated Flood Analysis System (IFAS) developed by International Center for Water Hazard and Risk Management (ICHARM), Public Works Research Institute of Japan (PWRI). The main objective of the IFAS is to reduce flood damages in developing countries where hydrologic information is not sufficiently available. In addition to satellitebased and ground-based rainfall data, Global Map data are used for runoff analysis. Global Map Land Use and Land Cover data are used for setting parameters, such as roughness and surface permeability in combination with other data, such as soil and geological data. Global Map Elevation data are used for creating a basin boundary and a water flow network.

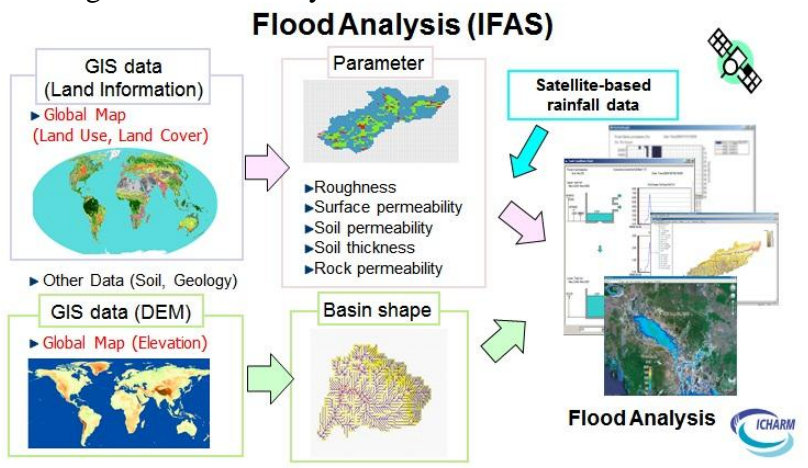

Figure 9: Flow of flood analysis in IFAS

Application in the field of Climate Change: Global Map can contribute to policy making processes in climate change field. Global Map can be used for the greenhouse gas (GHG) inventory in Land Use, Land-Use Change and Forestry (LULUCF) sector when the parties which contract United Nations Framework Convention on Climate Change (UNFCCC) report their national GHG inventories to the UNFCCC Secretariat based on UNFCCC and Kyoto Protocol. Global Map Land Cover data can be used for calculation of emissions and removals of GHGs as well as its supplementation and cross-checking, especially in the countries where statistics and geographic information are not well developed.

Global Map Land Cover data are introduced as an example of international land cover dataset in the Intergovernmental Panel on Climate Change (IPCC) Guidelines for GHG inventory: "IPCC Good Practice Guidance for LULUCF" and "2006 IPCC Guidelines for National Greenhouse Gas Inventories.”

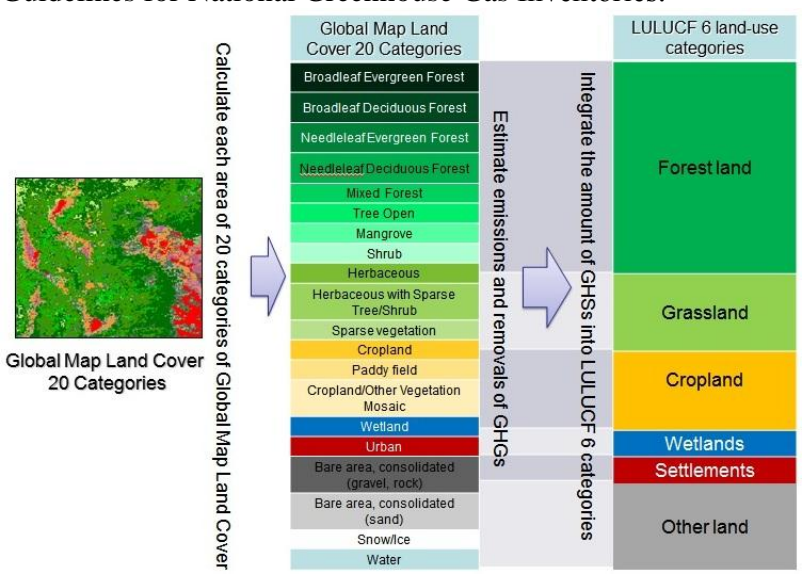

Figure 10: Application in GHG inventories

Application in the field of Biodiversity Protection: By overlaying the Global Map on data of wild life habitats, it is possible to visualize the spatial distribution of biodiversity, providing supports in biodiversity conservation.

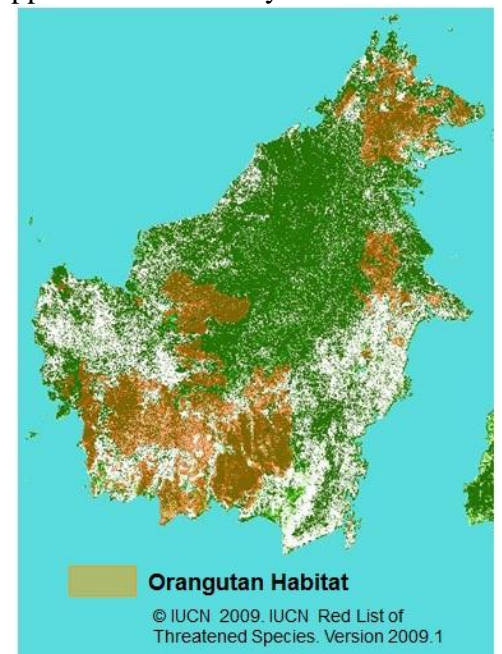

Figure 11: Global Map for biodiversity conservation planning

The 10th meeting of the Conference of the Parties of Convention on Biological Diversity (COP10) took place in Nagoya, Japan in October 2010. GSI displayed a large-scale Global Map poster ( $5 \mathrm{~m}$ by $3 \mathrm{~m}$ ) on the floor in the exhibition booth. The poster was created by overlaying habitats of threatened species on Global Map Land Cover.

Release of "Print-ready Global Map": The MLIT of Japan and the Secretariat of the ISCGM released "Print-ready Global Map" data via the Internet. This is a trial effort for the use of 
Global Map data without any GIS software. By adopting the Geospatial format, Global Map can be easily viewed with Adobe Reader which is available with no cost and virtually on all the PCs. The "Print-ready Global Map" data of all the Global Map data released before the time of the data conversion at the end of August 2011 are available at http://globalwebmap.jp/print_ready/english/.

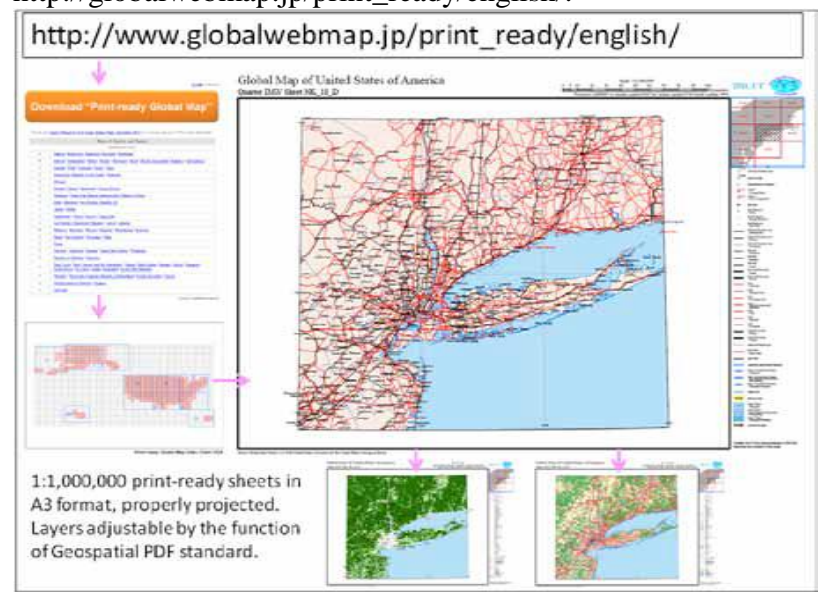

Figure 12: Release of "Print-ready Global Map"

\subsection{Analysis on the purpose of Global Map use}

The ISCGM Secretariat conducted a survey in 2011 to know the purpose of use of Global Map on the data-download site of the ISCGM website to be referred on the occasion of revision of the specifications for Version 3 planned in the near future. The purposes of downloading "national data", which are mostly vector data, are quite diverse. Major six purposes are Education (11\%), Earth Observation (10\%), Agriculture (9\%), Research study (8\%), Land Change (7\%) and Socioeconomics (7\%), but there are no prominent ones. Both uses of Global Land Cover and Vegetation are distributed in similar purposes. Earth observation is the largest for the both and account for $22 \%$ and $28 \%$, respectively. Research Study follows next, and Education and Land Change rank high.

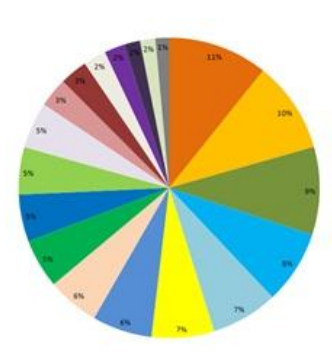

National Data (Vector data)

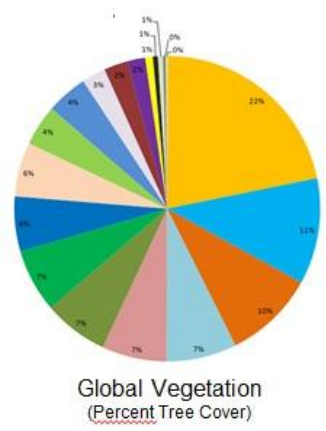

Figure 13: Purpose of data use

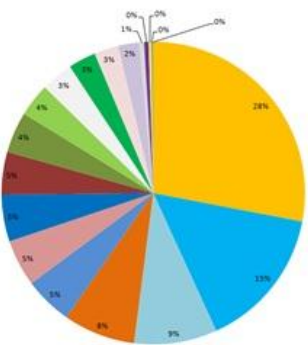

Global Land Cover

Education Earth Observation $=$ Agriculture

- Research stud

= Land Change

$\begin{aligned} & \text { Socioeconomics } \\ \text { - } & \text { Natural Resources }\end{aligned}$

Climate

- Water

- Ecosystems

= Geology

Disaster

Biodiversity

weather

Policy formulation

-Energy

- Health

- Telecommunications

- National Security

\section{CONCLUSION}

Needs for global geospatial Information: As observed in the establishment of the UN Committee of Experts on Global Geospatial Information Management, the need for the information for decision-making, including geospatial information, is still crucial for all people in the world even in the days of twenty years after UNCED held in Rio de Janeiro.

Progress of the Global Mapping Project: The Global Mapping Project is making steady progress to provide basic geospatial information developed in international cooperation since its launch in 1996 and the release of Global Map Version 1 in 2008. The ISCGM and all the project participating organizations make effort to develop high quality Global Map data and release the Global Map Version 2 in 2013.

Future prospect: In order to give more precise analysis on global issues, Global Map needs to be developed at a larger scale. In 2010, the ISCGM adopted a resolution asking for investigation of the secretariat into the necessity and feasibility of the development of Global Map at a larger scale. The ISCGM is now looking into specifications for the data development at 1:250,000 scale toward the Global Map Version 3.

The best use of high-resolution satellite images or remote sensing technology is the essential element to achieve these goals. The ISCGM and the project participating countries are going to make efforts to develop better Global Map and provide all people with the base of geospatial data infrastructure.

There are a lot of Global Map applications as well as potential applications. Further utilization of Global Map is expected.

\section{REFERENCES}

References from Journals:

Iimura, T., Nakamura, T., Otsuka, T., Ubukawa, T., Nakaminami, K., Motojima, Y., Suga, M. and Yatabe, Y., 2011. GSI's activities for the promotion of Global Map. Papers and Proceedings of the Geographic Information Systems Association, Vol. 20.

\section{References from Other Literature:}

Economic and Social Council, UN, 2011. Global Geospatial Information Management, Report of the Secretary General. Substantive session of 2011, E/2011/89.

Economic and Social Council, UN, 2011. United Nations Committee of Experts on Global Geospatial Information Management, Report of the first session. Economic and Social Council Official Records 2012.

International Steering Committee for Global Mapping. 2011, The Report of the eighteenth meeting of ISCGM (Winchester, U.K.). The Secretariat of ISCGM, Tsukuba, Japan

Kishimoto, N. Fukushima, Y., Tanaka, T., Nakamura, T., Otoi, K., Takahashi, H., Oomiya, S., Kojima, S. and Yoshikawa, M., 2009. Revision of Specifications for Global Map Version 2. Bulletin of the GSI, Vol. 57. 
International Archives of the Photogrammetry, Remote Sensing and Spatial Information Sciences, Volume XXXIX-B4, 2012 XXII ISPRS Congress, 25 August - 01 September 2012, Melbourne, Australia

\section{References from website:}

Fujimura, H., 2011. Release of "Print-ready Global Map". Global Mapping Newsletter, Vol. 65, http://www.iscgm.org/cgibin/fswiki/wiki.cgi?page=Newsletter $(10$ April 2012)

International Steering Committee for Global Mapping, 2011, Global Map Specification Version 2.1, http://www.iscgm.org/cgi-

bin/fswiki/wiki.cgi?page=Documentation (10 April 2012)

International Steering Committee for Global Mapping. 2012, ISCGM Website, http://www.iscgm.org/ (1 April 2012) 